\title{
Efficacy of colistin alone and in various combinations for the treatment of experimental osteomyelitis due to carbapenemase-producing Klebsiella pneumoniae
}

\author{
Anne-Claude Crémieux ${ }^{1,2 *}$, Aurélien Dinh ${ }^{2,3}$, Patrice Nordmann ${ }^{4}$, William Mouton ${ }^{5,6}$, Pierre Tattevin ${ }^{7,8}$, Idir Ghout ${ }^{9}$, \\ Aurelie Jayol ${ }^{4}$, Omar Aimer $^{10}$, Laure Gatin ${ }^{2}$, Marie-Clémence Verdier ${ }^{7,8}$, Azzam Saleh-Mghir ${ }^{2,3}$ and \\ Frédéric Laurent ${ }^{5,6}$
}

\begin{abstract}
${ }^{1}$ St Louis Hospital, Paris 7 University, Paris, France; ${ }^{2}$ UMR 1173, Versailles Saint-Quentin University, Versailles, France; ${ }^{3}$ Raymond Poincaré University Hospital, Garches, France; ${ }^{4}$ Medical and Molecular Microbiology Unit, Department of Medicine, Faculty of Science and Medicine, INSERM European Unit (IAME, France), Swiss National Reference Center for Emerging Antibiotic Resistance (NARA), University of Fribourg, Fribourg, Switzerland; ${ }^{5}$ Team 'Staphylococcal pathogenesis', International Centre for Infectiology Research, INSERM U1111 - CNRS UMR5308 - ENS Lyon - Lyon 1 University, Lyon, France; ${ }^{6}$ Institute for Infectious Agents, Department of Bacteriology - CNR des staphylocoques, Croix-Rousse Hospital, North Biology Centre, Hospices Civils de Lyon, Lyon, France;

${ }^{7}$ Pontchaillou University Hospital, Rennes, France; ${ }^{8}$ INSERM U1230, Rennes 1 University, IFR140, F-35033, Rennes, France; ${ }^{9}$ AP-HP, Ambroise Paré University Hospital, Boulogne, France; ${ }^{10}$ Department of Pharmacy, Raymond-Poincaré University Hospital, Garches, France
\end{abstract}

\footnotetext{
*Corresponding author. Maladies Infectieuses, Hôpital St Louis, 10 avenue Claude Vellefaux, 75010 Paris, France. Tel: +33-(1)-42-49-45-72; Fax: +33-(1)-42-49-48-20; E-mail: anne-claude.cremieux@aphp.fr
}

\begin{abstract}
Objectives: In a new experimental model of carbapenemase-producing Klebsiella pneumoniae osteomyelitis we evaluated the efficacy of colistin alone and in various combinations and examined the emergence of colistinresistant strains and cross-resistance to host defence peptides (HDPs).

Methods: KPC-99YC is a clinical strain with intermediate susceptibility to meropenem (MIC $=4 \mathrm{mg} / \mathrm{L})$ and full susceptibility to gentamicin, colistin and tigecycline (MICs $=1 \mathrm{mg} / \mathrm{L}$ ) and fosfomycin (MIC=32 mg/L). Time-kill curves were performed at $4 \times$ MIC. Osteomyelitis was induced in rabbits by tibial injection of $2 \times 10^{8} \mathrm{cfu}$. Treatment started 14 days later for 7 days in seven groups: (i) control; (ii) colistin; (iii) colistin + gentamicin; (iv) colistin + tigecycline; (v) colistin + meropenem; (vi) colistin + meropenem + gentamicin; and (vii) colistin + fosfomycin.
\end{abstract}

\begin{abstract}
Results: In vitro, colistin was rapidly bactericidal, but regrowth occurred after $9 \mathrm{~h}$. Combinations of colistin with meropenem or fosfomycin were synergistic, whereas combination with tigecycline was antagonistic. In vivo, colistin alone was not effective. Combinations of colistin with meropenem or fosfomycin were bactericidal $(P<0.001)$ and the addition of gentamicin enhanced the efficacy of colistin + meropenem $(P=0.025)$. Tigecycline reduced the efficacy of colistin $(P=0.007)$. Colistin-resistant strains emerged in all groups except colistin + fosfomycin and two strains showed cross-resistance to HDP LL-37.
\end{abstract}

Conclusions: In this model, combinations of colistin plus meropenem, with or without gentamicin, or colistin plus fosfomycin were the only effective therapies. The combination of colistin and tigecycline should be administered with caution, as it may be antagonistic in vitro and in vivo.

\section{Introduction}

The dramatic spread of carbapenemase-producing Enterobacteriaceae (CPE) is a global health problem and has been recently categorized by the WHO as a critical priority. ${ }^{1}$ These organisms are mostly responsible for urinary tract, bloodstream and surgical site infections and pneumonia in hospitalized patients. ${ }^{2}$ Posttraumatic or post-operative osteomyelitis and prosthetic joint infection (PJI) due to CPE have been more recently described in countries where CPE is endemic or in hospitals receiving patients from endemic areas. ${ }^{3,4}$ Treatment of these infections is especially difficult because they present a combination of the main current challenges in the field of antibiotics. First, despite the recent approval of new compounds, such as ceftazidime/ avibactam, therapeutic options remain scarce and could rely on 
old antibacterial agents with limited clinical data on their efficacy and a high risk of adverse events. Second, osteomyelitis is a difficult-to-treat infection because of the presence of biofilm ${ }^{5}$ and impaired diffusion of most antibiotics within bone tissues. ${ }^{6}$

Colistin is often one of the remaining options for carbapenemaseproducing Klebsiella pneumoniae. ${ }^{7,8}$ However, parenteral administration of colistin may be a source of adverse effects, including nephrotoxicity and neurotoxicity. ${ }^{7}$ Moreover, the emergence of colistin-resistant strains has been described in patients treated with colistin, ${ }^{9}$ possibly promoted by suboptimal drug exposure due to the low colistin therapeutic index, ${ }^{10,11}$ and this outcome may leave patients with no antibiotics left to treat lifethreatening infections, as recently reported by the CDC. ${ }^{12}$

Based mostly on cohort studies, experts recommend a combination of two active agents for the management of severe CPE infections, primarily colistin combined with meropenem if the MIC is $\leq 8 \mathrm{mg} / \mathrm{L}$ and/or an aminoglycoside, tigecycline or fosfomycin, based on in vitro susceptibility tests. ${ }^{13,14}$

Optimal treatment is poorly defined and it is critical to develop experimental models that closely reproduce human infections to provide direct comparisons of therapeutic regimens currently available for these difficult-to-treat infections with limited therapeutic options. Most models of CPE infections described in the literature are acute murine models of pneumonia, thigh infection or septicaemia. ${ }^{15-17}$ Our primary objective was to test the efficacy of various colistin-based combinations in a new subacute osteomyelitis model due to a KPC-producing K. pneumoniae.

In addition, given that (i) cross-resistance to colistin and host defence peptides (HDPs) LL-37 and lysozyme has been previously described $^{18}$ and (ii) we previously demonstrated the emergence of cross-resistance to HDPs and daptomycin in an experimental model of PJI in rabbits, ${ }^{19,20}$ we investigated the emergence of colistin-resistant strains in untreated and treated rabbits with osteomyelitis and cross-resistance to HDPs.

\section{Materials and methods}

\section{Bacterial strain}

K. pneumoniae KPC-99YC is a clinical strain with intermediate susceptibility to meropenem ( $\mathrm{MIC}=4 \mathrm{mg} / \mathrm{L}$ ) and full susceptibility to gentamicin, colistin and tigecycline (MICs=1 mg/L) and fosfomycin ( $\mathrm{MIC}=32 \mathrm{mg} / \mathrm{L})$. This strain produces $\mathrm{KPC}-2$ carbapenemase and belongs to the epidemic clone ST-258.

Before induction of the animal model of infection, the KPC-producing strain was cultured in casein hydrolysate-yeast extract-containing (CCY) broth medium at $37^{\circ} \mathrm{C}$ for $18 \mathrm{~h}$. After centrifugation, the pellets were washed twice and resuspended in PBS before inoculation. All inocula were quantified by plating serial dilutions on tryptic soy agar (bioMérieux, La Balme-les-Grottes, France).

\section{Time-kill curve studies}

The bactericidal activities of colistin, meropenem, gentamicin and fosfomycin, alone or in combination, were determined in duplicate or triplicate. Overnight cultures were diluted in $10 \mathrm{~mL}$ of fresh Mueller-Hinton broth to yield an inoculum of $\sim 10^{5} \mathrm{cfu} / \mathrm{mL}$ as often recommended for macrodilution time-kill assays and corresponding to the bacterial load in bones. ${ }^{21-23}$ The antibiotic concentrations used were equivalent to $4 \times$ MIC. ${ }^{24}$ After $0,3,6,9$ and $24 \mathrm{~h}$ of incubation in a shaking water bath at $37^{\circ} \mathrm{C}$, serial dilutions of $0.1 \mathrm{~mL}$ samples were subcultured on Mueller-Hinton agar plates (Bio-Rad,
Marnes la Coquettes, France) and incubated at $37^{\circ} \mathrm{C}$ for $24 \mathrm{~h}$ before the numbers of cfu were counted. A bactericidal effect was defined as a $>3 \log _{10}$ cfu decrease compared with the initial inoculum. Synergy was defined as a decrease of $>2 \log _{10} \mathrm{cfu} / \mathrm{mL}$ for the combination compared with that of its most active constituent. Antagonism was defined as an increase of $>2 \log _{10} \mathrm{cfu} / \mathrm{mL}$ for the combination compared with that of its most active component.

\section{Selection of antibiotic doses in rabbits}

Plasma antibiotic concentrations were measured in uninfected rabbits to select doses leading to plasma concentrations equivalent to those obtained in humans. Each antibiotic was tested on one or several groups of four uninfected rabbits ( $n=16$ for colistin and 12 for meropenem). After $24 \mathrm{~h}$ of treatment (steady-state concentrations), blood samples were drawn $15 \mathrm{~min}$ and $1,2,4,6,8$ and $12 \mathrm{~h}$ after the injection and centrifuged, and plasma was frozen until assayed.

Gentamicin concentrations were determined using an enzyme immunoassay, kinetic interaction of microparticles in solution (KIMS), on a Roche Cobas system (Roche Diagnostics, Mannheim, Germany). Meropenem, fosfomycin, tigecycline and colistin were analysed by HPLCtandem MS using an electrospray ionization method. For meropenem, fosfomycin and tigecycline, the HPLC system was interfaced with a triplestage quadrupole spectrometer Finnigan TSQ Quantum Discovery Max from Thermo Fisher (Waltham, USA). Reversed-phase chromatography was performed on an Atlantis ${ }^{\circledR} \mathrm{T} 3 \mathrm{dC} 18$ column $(3 \mu \mathrm{m}, 2.1 \times 100 \mathrm{~mm}$; Waters, Milford, USA). The lower limits of quantification were 1, 2 and $0.25 \mathrm{mg} / \mathrm{L}$ for meropenem, fosfomycin and tigecycline, respectively. Colistin was analysed using a triple-stage quadrupole spectrometer Acquity H Class-Quattro Premier XE from Waters. The samples were purified by solid-phase extraction on OASIS HLB Cartridges (Waters) and separation was performed on a $C_{18}$ XBridge $^{T M}$ column $(3.5 \mu \mathrm{m}, 2.1 \times 100 \mathrm{~mm}$; Waters). The transitions of the $[\mathrm{M}+3 \mathrm{H}]^{3+}$ precursors to the product ions were $\mathrm{m} / \mathrm{z} 390.82>100.73 \mathrm{~m} / \mathrm{z} 386.18>100.73$ and $\mathrm{m} / \mathrm{z} 402.16>100.85$ for colistin A, colistin B and polymyxin B, respectively. The concentration range was 62.5 to $8000 \mathrm{ng} / \mathrm{mL}$ for colistins A + B.

Pharmacokinetic parameters were calculated using Monolix version 2016R1 (Lixoft SAS, Antony, France).

\section{Rabbit osteomyelitis model}

The method of Norden ${ }^{25}$ was used to induce osteomyelitis in female New Zealand white rabbits, each weighing 2 to $3 \mathrm{~kg}$. The rabbits were housed in individual cages and received food and water ad libitum. The experimental protocol complied with French legislation on animal experimentation and was approved by the Animal Use Committee of Maisons-Alfort Veterinary School. The animals were anaesthetized by intramuscular (im) injection of ketamine $(25 \mathrm{mg} / \mathrm{kg}$; Vibrac, Carros, France) and $2 \%$ xylazine $(25 \mathrm{mg} / \mathrm{kg}$; Bayer Santé, Puteaux, France). Infection was induced by tibial intramedullary injection of a sclerosing agent, $0.1 \mathrm{~mL}$ of $3 \%$ sodium tetradecyl sulphate (Kreussler Pharma, La Chaussée-Saint-Victor, France), followed by $0.2 \mathrm{~mL}$ of a KPC-99YC inoculum $\left(10^{9} \mathrm{cfu} / \mathrm{mL}\right)$ and $0.1 \mathrm{~mL}$ of saline. Patch analgesia with fentanyl (Janssen-Cilag, Issy-les Moulineaux, France) was given for 7 days following surgery. A $10^{9} \mathrm{cfu} / \mathrm{mL}$ inoculum was selected because preliminary experiments (data not shown) revealed that it induced persistent osteomyelitis in $90 \%$ of animals, with low sepsis-related early mortality $(<10 \%)$.

\section{Treatment and its evaluation}

Rabbits were randomly assigned to one of seven groups of 12 rabbits: (i) control; (ii) colistin $150000 \mathrm{IU} / \mathrm{kg}$ q8h im (equivalent to 3 million IU q8h in humans); (iii) colistin + gentamicin $30 \mathrm{mg} / \mathrm{kg}$ im q24h (5 mg/kg q24h in humans); (iv) colistin + tigecycline $14 \mathrm{mg} / \mathrm{kg}$ im q12h (50 mg q12h in humans); (v) colistin + meropenem $80 \mathrm{mg} / \mathrm{kg}$ subcutaneously (sc) q8h ( $250 \mathrm{mg}$ q8h in humans); (vi) colistin + meropenem + gentamicin; and (vii) colistin + fosfomycin 
$150 \mathrm{mg} / \mathrm{kg} \mathrm{q12h}$ im (4 g q8h in humans). The same dose of colistin was used in all groups. Each regimen was started 14 days after surgery and administered for 7 days. Rabbits with no osteomyelitis and those that died before surgery, due to anaesthesia, or before the start of treatment were not included (Table S1, available as Supplementary data at JAC Online).

Rabbits were euthanized by intravenous (iv) injection of pentobarbital 3 days after the end of therapy (day 24 post-infection) to allow for bacterial regrowth after treatment discontinuation. Control rabbits were also euthanized on day 24. At the time of death, the upper third of the tibia $(3 \mathrm{~cm}$ long), including compact bone and marrow, was isolated, split with a bone crusher, weighed, cut into small pieces, frozen in liquid nitrogen and crushed in an autopulverizer (Spex 6700; Freezer/Mill Industries Inc., Metuchen, USA). The pulverized bone was suspended in $10 \mathrm{~mL}$ of sterile saline. Serial dilutions were made and plated on tryptic soy agar. After $24 \mathrm{~h}$ of incubation at $37^{\circ} \mathrm{C}$, the number of viable microorganisms was determined. The results were expressed as the median (IQR) $\log _{10} \mathrm{cfu} / \mathrm{g}$ of bone and as the percentage of animals with sterile bones. Bone was considered sterile when the culture showed no growth after incubation for $48 \mathrm{~h}$ at $37^{\circ} \mathrm{C}$ and the number of cfu was recorded as the lowest detectable bacterial count (1.10 to $1.30 \mathrm{cfu} / \mathrm{g}$ of bone, depending on the weight of the sample). Analyses were performed including all animals that received at least one dose of the treatment assigned.

\section{In vivo selection of mutants}

Each undiluted bone homogenate $(50 \mu \mathrm{L})$ of untreated and treated rabbits was plated onto Mueller-Hinton II agar and onto Mueller-Hinton II agar supplemented with colistin $(0.125,0.25,0.5,1,2,4$ or $16 \mathrm{mg} / \mathrm{L})$ to detect resistant mutants after $24 \mathrm{~h}$ of incubation at $37^{\circ} \mathrm{C}$. When bacterial growth was observed, K. pneumoniae identification was confirmed using MALDITOF MS (VITEK MS; bioMérieux, La Balme-les-Grottes, France). The MIC values of colistin were determined by liquid broth dilution methods based on the UMIC kit (Biocentric, Bandol, France). Resistant mutants were defined by an MIC value $>2 \mathrm{mg} / \mathrm{L}$ (i.e. MIC increased by at least 2 -fold compared with that for the initial strain).

\section{Genetic characterization of colistin-resistant strains}

Mgr protein is involved in the regulation of LPS biosynthesis in K. pneumoniae. ${ }^{26}$ Mutations in the mgrB gene are key elements as a source of acquired resistance to colistin in K. pneumoniae. ${ }^{27}$ Therefore, alterations in the $\mathrm{mgrB}$ gene sequences were sought in colistin-resistant strains that emerged in control or treated rabbits. The $\mathrm{mgrB}$ genes of four colistinresistant isolates were amplified with pre-mgrBF and mgrB-extR primers and sequenced as reported. ${ }^{27}$

\section{Cross-resistance to human antimicrobial peptides (AMPs)}

Colistin-resistant bacteria were tested for their susceptibility to specific AMPs produced by human cells in response to infections or wounds and by infiltrating neutrophils: LL-37, human $\alpha$-defensin 5 (HDA5) and human $\beta$ defensin 3 (HDB3). ${ }^{26}$

Approximately $1000 \mathrm{cfu}$ of mid-logarithmic phase bacteria were incubated with $0,0.3125,0.625,1.25,2.5,5,10$ and $20 \mathrm{mg} / \mathrm{L}$ diluted peptide for $2 \mathrm{~h}$ in a 96 -well plate at $37^{\circ} \mathrm{C}$ in PBS supplemented with $1 \% \mathrm{LB}$ broth. The upper limit of $20 \mathrm{mg} / \mathrm{L}$ peptide was based on previous studies, ${ }^{28,29}$ which showed less than $20 \%$ survival at this concentration. Samples were plated in triplicate on LB agar plates to quantify the remaining bacteria by cfu counts and the assays were run at least in duplicate for each peptide concentration. The means and standard deviations were calculated for each condition.

\section{Data analysis}

The data were analysed with $\mathrm{R}$ software. ${ }^{30}$ Due to the small sample size and asymmetric distributions of variables, exact non-parametric tests implemented in the coin package were used. ${ }^{31}$ Data of numeric variables were summarized by the minimum, maximum, median, range, IQR, mean and standard deviation, and data of categorical variables by numbers and percentages. Between-group comparisons were performed using appropriate statistical tests. The $\chi^{2}$ test, Fisher's exact test and the Kruskal-Wallis non-parametric method were used. The effect of antibiotics and their $95 \%$ CIs on $\log _{10}$ cfu criteria were calculated by the Hodges-Lehmann estimator and the Bauer algorithm, respectively. The effect of the antibiotics on the sterility criteria was estimated by the difference in the proportion of sterile bones and the $95 \%$ exact CI. A $P$ value $<0.05$ was considered statistically significant.

\section{Results}

\section{In vitro bactericidal effect}

Time-kill curves obtained at $4 \times$ MIC (Figure 1 ) showed that colistin alone was rapidly bactericidal during the first $6 \mathrm{~h}$. However, regrowth occurred after $9 \mathrm{~h}$ of incubation. The MICs for isolates obtained after regrowth were similar to the MICs for the initial strain. Meropenem alone was slowly bactericidal, with a decrease in the initial inoculum by only $2 \log _{10}$ at $9 \mathrm{~h}$. Fosfomycin and gentamicin alone exhibited early bactericidal activity, but regrowth was observed at 3 and $6 \mathrm{~h}$, respectively.

For the combination of gentamicin and colistin, rapid bactericidal activity was observed (with a complete bactericidal effect up to $2 \mathrm{~h}$ ), but regrowth was observed after $3 \mathrm{~h}$. When fosfomycin was added to colistin, rapid bactericidal activity was observed (as with colistin alone). However, this combination of antibiotics prevented regrowth. The combination of colistin, meropenem and gentamicin was the most effective combination, with a fast and complete bactericidal effect at $3 \mathrm{~h}$ and no subsequent regrowth.

Tigecycline alone was not bactericidal and slight regrowth was observed at $9 \mathrm{~h}$ (Figure 2). The combination of tigecycline and colistin was antagonistic, with a loss of the initial bactericidal effect observed with colistin alone. A dose-dependent decrease of bactericidal activity for the combination was observed when a range of tigecycline concentrations (2-fold serial dilutions, from 32 to $4 \mathrm{mg} / \mathrm{L})$ with a fixed concentration of colistin $(4 \times \mathrm{MIC})$ were tested (data not shown).

\section{Serum concentrations of meropenem and colistin in rabbits}

For colistin, the pharmacokinetic/pharmacodynamic target (AUC/ MIC ratio of 25-50) was achieved with the dosing regimen of $150000 \mathrm{IU}$ three times daily im, equivalent to $3 \mathrm{M}$ IU q8h in humans. For meropenem, the initial target ( $T_{>\text {MIC }} 50 \%$ ) was achieved with $250 \mathrm{mg} / \mathrm{kg}$ q $8 \mathrm{~h}$, but the treatment induced severe diarrhoea and lethality of $>50 \%$. With a dosage of $80 \mathrm{mg} / \mathrm{kg} \mathrm{q} 8 \mathrm{~h}$ SC ( $T_{>\text {MIC }} 20 \%$ ), the plasma mean $C_{\max }$ and mean $C_{\min }$ were $17.9 \pm 12.4$ and $0.35 \pm 0.11 \mathrm{mg} / \mathrm{L}$, respectively. These values were comparable to the $C_{\max }$ and $C_{\min }$ values observed with $250 \mathrm{mg}$ $\mathrm{q} 8 \mathrm{~h}$ in humans. For gentamicin, the pharmacokinetic/pharmacodynamic parameter target $\left(C_{\max }=10 \times \mathrm{MIC}\right)$ was achieved with a dosage of $30 \mathrm{mg} / \mathrm{kg}$ q $24 \mathrm{~h} \mathrm{im}$. For fosfomycin, $C_{\max }$ and AUC values of $400 \mathrm{mg} / \mathrm{L}$ and $1128 \mathrm{mg} \cdot \mathrm{h} / \mathrm{L}$, equivalent to $4 \mathrm{~g}$ q8h in humans and allowing a $T_{>\text {MIC }}$ of $>50 \%$, were obtained with $150 \mathrm{mg} / \mathrm{kg}$ $\mathrm{q} 12 \mathrm{~h}$ im dosage. For tigecycline, the pharmacokineticl 
(a)

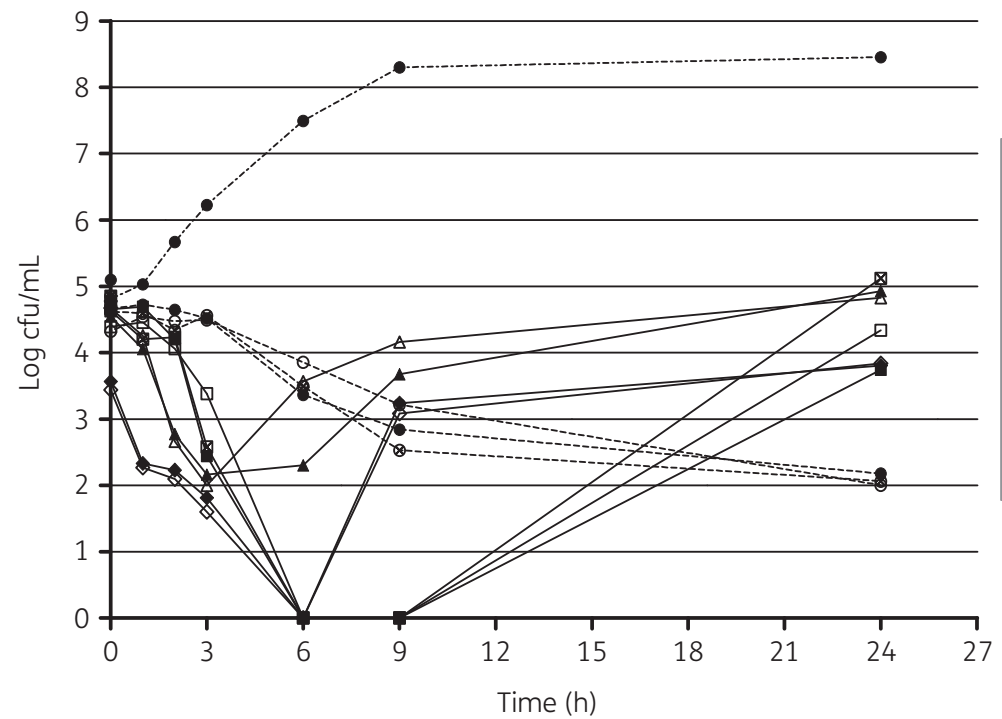

--- Control

$\rightarrow$ Colistin experiment 1

$\because$ Colistin experiment 2

$\rightarrow-$ Colistin experiment 3

- $\bullet$ Meropenem experiment 1

- - Meropenem experiment 2

- - - Meropenem experiment 3

$\multimap$ Gentamicin experiment 1

$\diamond$ Gentamicin experiment 2

$₫$ Fosfomycin experiment 1

$\triangle$ Fosfomycin experiment 1

(b)

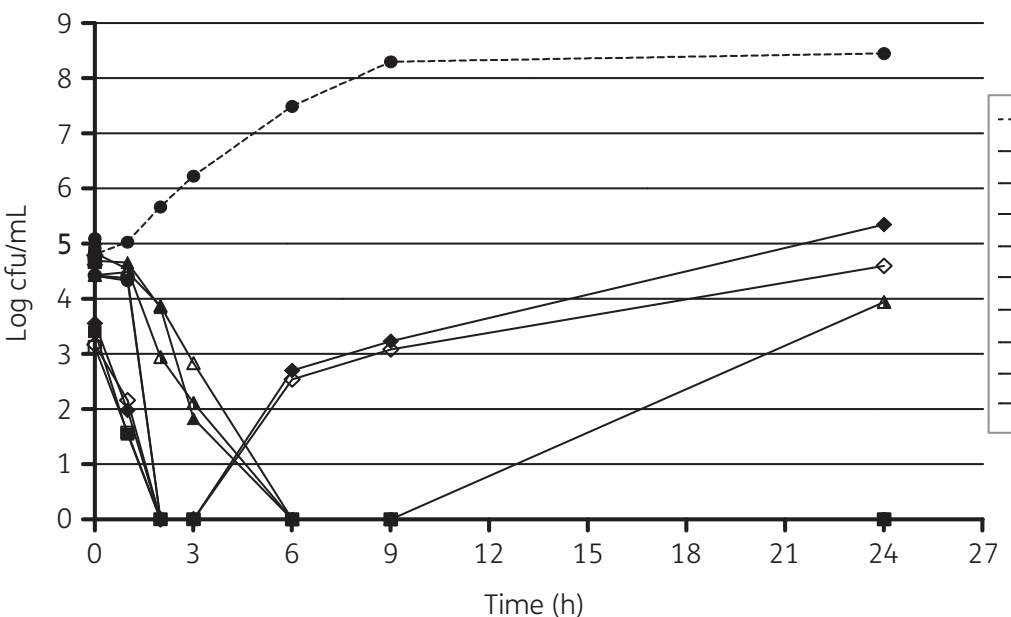

\section{- - Control}

- Colistin+fosfomycin experiment 1

o- Colistin+fosfomycin experiment 2

- Colistin+gentamicin experiment 1

$\diamond$ - Colistin+gentamicin experiment 2

$\leftarrow$ Colistin+meropenem experiment 1

$\triangle$ Colistin+meropenem experiment 2

$\triangle \mathbf{A}$ Colistin+meropenem experiment 3

$\rightarrow-$ Colistin+meropenem+gentamicin experiment 1

$\square$ Colistin+meropenem+gentamicin experiment 2

Time (h)

Figure 1. In vitro killing curves for carbapenemase-producing K. pneumoniae strain KPC-99YC using various antibiotics alone (a) or in combination (b) at concentrations equivalent to $4 \times$ MIC. Experiments were performed in duplicate or triplicate.

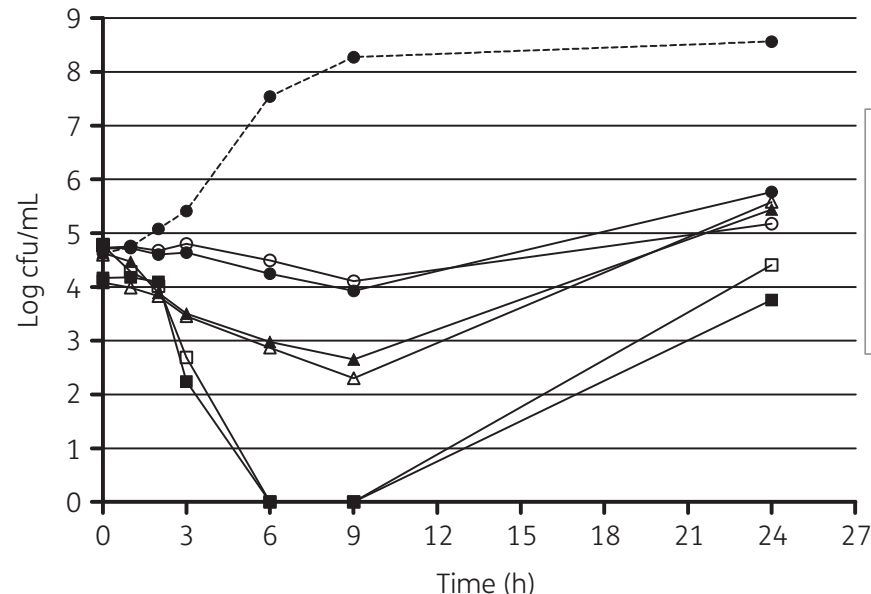

\section{---- Control}

$\rightarrow$ Colistin experiment 1

$\square$ Colistin experiment 2

$\rightarrow$ Tigecycline experiment 1

- Tigecycline experiment 2

$\rightarrow$ Colistin+tigecycline experiment 1

$\triangle$ Colistin+tigecycline experiment 2

Time $(h)$

Figure 2. In vitro killing curves for carbapenemase-producing K. pneumoniae strain KPC-99YC using colistin and tigecycline alone and in combination at concentrations equivalent to $4 \times$ MIC. Experiments were performed in duplicate. 
(a)

Groups

N Median $\log _{10} \mathrm{cfu}$ (IQR)

Effect size (95\% CI)

$P$

Bacterial load reduction in $\log _{10} \mathrm{cfu}$

Control

$136(5.75,6.44)$

Colistin alone

$11 \quad 5.5(4.29,6.15)$

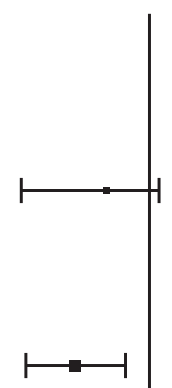

$-0.61(-1.81,0.14)$

0.106

Colistin + gentamicin

$114.99(4.63,5.6)$

$11 \quad 6.58(6,7.25)$

Colistin + tigecycline

$12 \quad 4.55(3.91,5.25)$

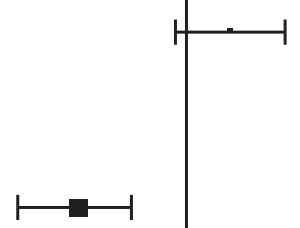

$-1.53(-2.38,-0.78)$

$<0.001$

Colistin + meropenem

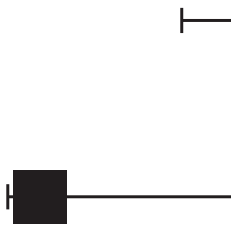

$-1.04(-1.75,-0.34)$

0.003

10

$1.48(1.45,3.98)$

$0.62(-0.16,1.39)$

0.096

$\frac{0}{0}$

Colistin + fosfomycin

$11 \quad 3.97(3.22,4.6)$

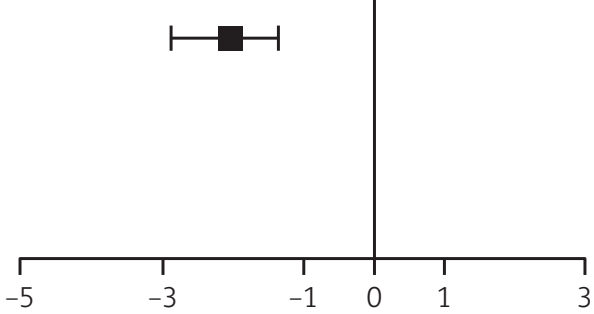

$-2.03(-2.88,-1.36)<0.001$

In favour of treatment

In favour of control

Figure 3. Effect of colistin alone or in combination with various antibiotics on $\log _{10} \mathrm{cfu} / \mathrm{g}$ of bone (a) and on percentage of sterile bones (b) in animals with carbapenemase-producing K. pneumoniae (strain KPC-99YC) experimental osteomyelitis.

pharmacodynamic target (AUC/MIC ratio >18) was achieved with a dosage of $14 \mathrm{mg} / \mathrm{kg} \mathrm{q} 12 \mathrm{~h} \mathrm{im}$.

\section{Therapeutic studies}

In control rabbits at day 24 , the median (IQR) of bacterial counts was 6 (5.8-6.4) $\log _{10}$ cfu/g of bone.

Compared with those of controls (Figure 3a and Figure 4), bacterial counts in rabbits treated with colistin alone were not different, with a median (IQR) of $5.5(4.3-6.2) \log _{10} \mathrm{cfu} / \mathrm{g}$ of bone $(P=0.106)$ and no sterile bones at day 24 (Figure 3b). Colistin combined with meropenem or gentamicin or fosfomycin or combined with meropenem + gentamicin significantly decreased bone bacterial concentrations. In accordance with time-kill curves, the triple combination of colistin plus meropenem plus gentamicin was more effective than colistin plus meropenem and colistin plus gentamicin on bone bacterial density (Table 1) and was the only effective regimen for bone sterilization (Figure 3b). Notably, as observed in vitro with time-kill curves, an antagonist effect was observed with colistin plus tigecycline, which was significantly less effective than colistin alone on bone bacterial concentrations $(P=0.007)$ (Table 1 ). 
(b)

\begin{tabular}{|c|c|c|c|c|c|c|}
\hline \multirow[t]{2}{*}{ Groups } & \multirow[t]{2}{*}{ N } & \multirow{2}{*}{\multicolumn{2}{|c|}{ Sterile bone (\%) }} & \multicolumn{2}{|l|}{ Effect size (95\% CI) } & \multirow[t]{2}{*}{$P$} \\
\hline & & & & \multicolumn{2}{|c|}{ Difference between proportion of sterile bones } & \\
\hline Control & 13 & 0 & & & - & - \\
\hline Colistin alone & 11 & 0 & $\longmapsto$ & & $0(-24.7,28.5)$ & $>0.999$ \\
\hline Colistin + gentamicin & 11 & $1(9.1)$ & $\longmapsto$ & $\longrightarrow$ & $9.1(-15.6,41.3)$ & 0.458 \\
\hline Colistin + tigecycline & 11 & 0 & $\longmapsto$ & $\longrightarrow$ & $0(-24.7,28.5)$ & $>0.999$ \\
\hline Colistin + meropenem & 12 & $1(8.3)$ & $\longmapsto$ & $\longrightarrow$ & $8.3(-16.2,38.5)$ & 0.48 \\
\hline Colistin + meropenem + gentamicin & 10 & $7(70)$ & & r & $70(33.8,93.6)$ & $<0.001$ \\
\hline Colistin + fosfomycin & 11 & 0 & $\longmapsto$ & $\longrightarrow$ & $0(-24.7,28.5)$ & $>0.999$ \\
\hline & & -50 & & 50 & & \\
\hline
\end{tabular}

Figure 3. Continued.

\section{Colistin-resistant mutants}

Colistin-resistant mutants emerged in rabbits treated with colistin $[n=2 ; \mathrm{L} 31$ and L35 (MICs=64 mg/L)], colistin + gentamicin [ $n=3$; L37, L43 and L48 (MIC=64, 64 and $16 \mathrm{mg} / \mathrm{L}$, respectively)], colistin + tigecycline $[n=2 ; \mathrm{L} 57(\mathrm{MIC}=16 \mathrm{mg} / \mathrm{L})$ and $\mathrm{L} 59(\mathrm{MIC}=64 \mathrm{mg} / \mathrm{L})]$ and colistin + meropenem + gentamicin $[n=1 ; \mathrm{L} 74(\mathrm{MIC}=32 \mathrm{mg} / \mathrm{L})]$. Notably, colistin-resistant strains were also detected in one untreated rabbit at day 24 [L17 (MIC=8 mg/L)].

\section{Characterization of colistin-resistant strains}

Mutation in the mgrB gene

As colistin resistance was unstable in vitro, selected colistinresistant strains were subcultured on Mueller-Hinton II agar with $2 \mathrm{mg} / \mathrm{L}$ colistin before amplification of the $\mathrm{mgrB}$ gene by PCR and sequencing. MgrB proteins from mutant L35 (from a rabbit treated with colistin alone) and mutant L59 (from a rabbit treated with colistin + tigecycline) were truncated. For strain L35, the truncation was due to insertion of an ISKpn26-like sequence in the coding sequence of the gene, whereas for strain L59 the truncation was due to a nucelotide substitution responsible for a premature stop codon (Table 2). For strain L37 (from a rabbit treated with colistin + gentamicin), no amplicon was obtained with mgrB primers, suggesting either a complete deletion of the mgrB gene or mutations in regions corresponding to the binding of the primers used for amplification of the mgrB gene (Table 2). Finally, the mgrB gene was partially deleted in the L17 control rabbit. Other resistant strains (L31 and L57) had a WT mgrB gene, suggesting other chromosomally encoded resistance mechanisms (Table 2).

\section{Cross-resistance to host AMPS}

Colistin-resistant strains that emerged in control rabbits [L17 ( $M I C=8 \mathrm{mg} / \mathrm{L})$ ] had a slightly increased resistance to LL-37 with decreased susceptibility at $10 \mathrm{mg} / \mathrm{L}$ compared with the initial strain 


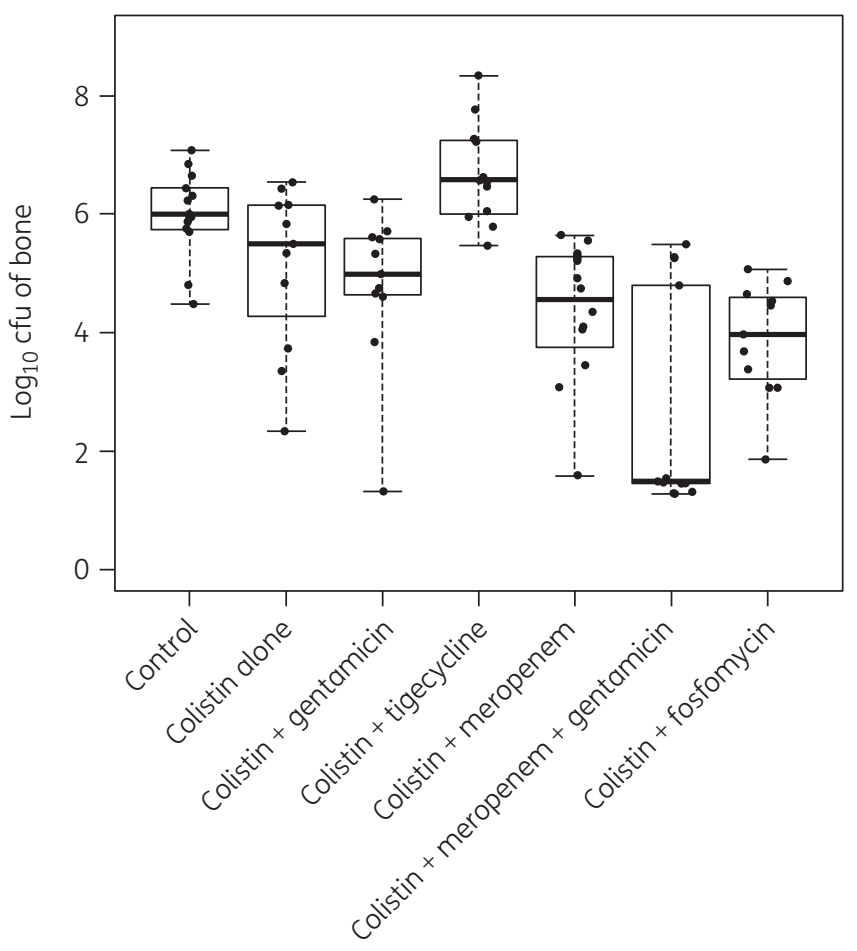

Figure 4. Bacterial density ( $\log _{10} \mathrm{cfu} / \mathrm{g}$ of bone) of colistin alone or in combination with various antibiotics for the treatment of carbapenemase-producing K. pneumoniae experimental osteomyelitis due to strain KPC-99YC. Whiskers are the minimum and the maximum values and the horizontal line in each box plot, which covers the IQR, is the median.

Table 1. Pairwise comparisons of colistin in combination with various antibiotics in carbapenemase-producing K. pneumoniae (strain KPC-99YC) experimental osteomyelitis

\begin{tabular}{lcc}
\hline Antibiotics & $\begin{array}{c}\text { Difference in } \\
\log _{10} \text { cfu }(95 \% \mathrm{CI})\end{array}$ & $P$ \\
\hline Colistin + meropenem + & & \\
gentamicin compared with: & & \\
colistin + gentamicin & $-3.2(-4.07$ to -0.09$)$ & 0.024 \\
colistin + tigecycline & $-4.58(-5.73$ to -2.43$)$ & $<0.001$ \\
colistin + meropenem & $-2.58(-3.61$ to -0.1$)$ & 0.025 \\
colistin + fosfomycin & $-1.86(-3.07-0.34)$ & 0.095 \\
Colistin + gentamicin compared & & \\
with: & & \\
colistin + tigecycline & $-1.63(-2.63$ to -0.87$)$ & $<0.001$ \\
colistin + meropenem & $0.5(-0.32-1.49)$ & 0.267 \\
colistin + fosfomycin & $1.04(0.14-1.92)$ & 0.019 \\
Colistin + tigecycline compared & & \\
with: & & $<0.001$ \\
colistin + meropenem & $2.23(1.28-3.17)$ & $<.001$ \\
colistin + fosfomycin & $2.72(1.93-3.59)$ & $<.007$ \\
colistin alone & $1.24(0.33-2.6)$ & \\
Colistin + meropenem compared & & 0.184 \\
with: & &
\end{tabular}

(Figure 5). HDB3 and HDA5 showed lower toxicity, as previously observed, and no difference was observed between the parental strain KPC-99YC and the colistin-resistant KPC-99YC derivatives (Figure 5). The colistin-resistant strain L31, with a colistin MIC of $64 \mathrm{mg} / \mathrm{L}$, that emerged in a rabbit with osteomyelitis treated by colistin alone was not susceptible to LL-37 even at a high concentration, in contrast to the initial parental strain (Figure 5).

\section{Discussion}

The efficacy of colistin in bone infections due to Gram-negative bacilli is poorly known. Our work showed that the efficacy of colistin alone or in combination, in a new subacute stringent model of KPC-producing K. pneumoniae osteomyelitis, was limited. Monotherapy with colistin was ineffective and colistin-resistant strains emerged after 14 days of treatment. The addition of gentamicin, fosfomycin or meropenem was the only effective therapy. The triple combination of colistin + meropenem + gentamicin was the only regimen able to sterilize most animals. However, colistinresistant strains emerged in all groups, except in rabbits treated with colistin + fosfomycin. Notably, the suppression of colistinresistant strains by fosfomycin has been described in time-kill experiments by others and this effect could be due to the killing of colistin-resistant strains by fosfomycin. ${ }^{32}$

The findings regarding osteomyelitis are in line with clinical observations and with recommendations for the treatment of other CPE infections. ${ }^{14,33}$ Indeed, those expert recommendations, based mostly on retrospective or prospective non-comparative clinical studies, state that: (i) colistin should always be used in combination with other drug(s); and (ii) penems should be included in the combination when the MIC is $\leq 8 \mathrm{mg} / \mathrm{L}$.

The combination of colistin and tigecycline is often used for treating severe KPC-producing K. pneumoniae infections, as tigecycline often remains active in vitro. Notably, our data highlighted that this combination was less bactericidal against KPC-99YC than colistin alone, suggesting an antagonistic effect in vivo. These data are in agreement with those provided by in vitro time-kill curves with KPC99YC. The antagonistic effect of the tigecycline + colistin combination has already been described in vitro with other CPE, including NDM1-producing Enterobacteriaceae, ${ }^{34}$ and in vivo in a thigh infection model of KPC-producing Enterobacteriaceae. ${ }^{35}$ This may be due to the mechanisms of action of tigecycline and colistin, i.e. inhibition of protein synthesis, interaction with LPS and DNA biosynthesis, which may be antagonistic. The experiments performed with two other KPC-producing K. pneumoniae (data not shown) belonging to distinct clonal complexes suggested that the antagonist effect may be strain-dependent and deserves to be tested when a clinical strain is isolated before the use of such a combination.

An interesting finding of this work is that our in vivo results mirror in vitro time-kill curves performed with logarithmic-growth phase bacteria. This result, which was also found by others in a foreign-body experimental model due to ESBL-Escherichia coli, ${ }^{36}$ contrasts with results obtained with MRSA experimental implantrelated infections, where the in vivo efficacy of antibiotics on MRSA is correlated with the bactericidal activity of antibiotics on stationary-growth phase bacteria. ${ }^{37}$ This finding suggests that in subacute infections such as osteomyelitis, the in vivo adaptation of the organisms to the local environment depends on the bacterial 
Table 2. Analysis of the mgrB gene sequences in the parental KPC-99YC strain that is susceptible to colistin inoculated into rabbits and some colistin-resistant strains that emerged in controls and treated rabbits with KPC-99YC osteomyelitis sacrificed 24 days post-infection

\begin{tabular}{lccc}
\hline Isolate & Treatment & Colistin MIC (mg/L) & mgrB gene \\
\hline KPC-99YC parental strain & none (control) & 0.25 & WT \\
L17 & colistin & 8 & partial deletion \\
L31 & colistin & 64 & WT \\
L35 & 64 & & insertion of an ISKpn26-like sequence into the \\
& & 64 & coding sequence \\
L37 & colistin + gentamicin & 16 & total deletion \\
L57 & colistin + tigecycline & 64 & WT \\
L59 & colistin + tigecycline & & truncated (stop codon)
\end{tabular}

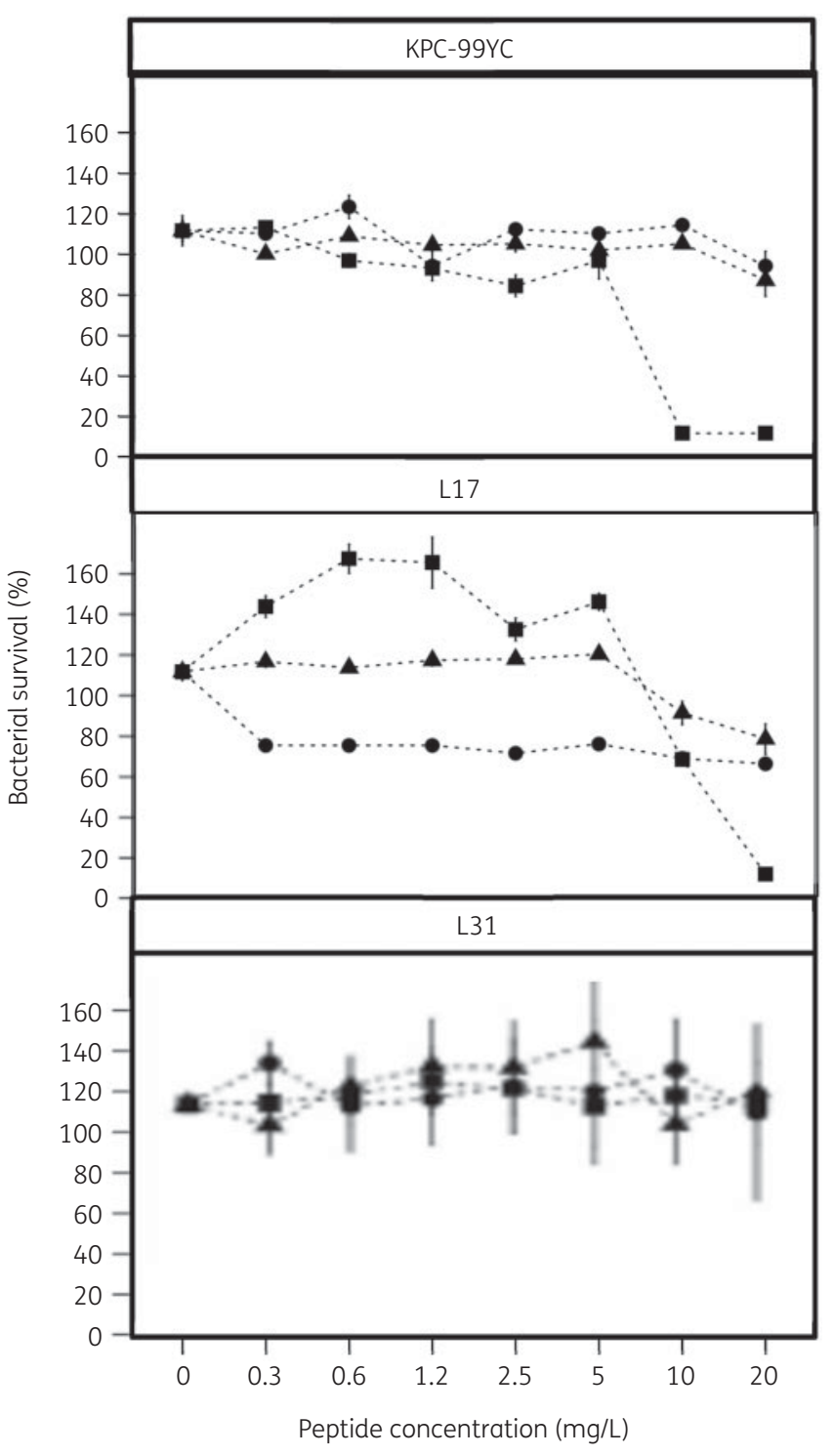

Figure 5. Percentage of bacterial survival as a function of peptide concentration. Panels show data for the initial strain (KPC-99YC) and colistin-resistant K. pneumoniae strains. Data represent the experimental means (symbols) \pm standard errors of the mean (vertical bars) for cationic AMPs LL-37 (squares), HDA5 (circles) and HDB3 (triangles). species. The concordance between in vitro and in vivo results, as well as the strain-dependent antagonistic effect of tigecycline combined with colistin, suggests that in vitro time-kill curves could be a useful tool to select the best therapeutic combination for severe KPC-producing K. pneumoniae infections.

Finally, we documented the treatment-induced emergence of colistin-resistant strains. As already described for several K. pneumoniae strains from humans treated with colistin, ${ }^{27}$ this colistin resistance trait was mostly due to mutation/deletion in the mgrB gene involved in LPS biosynthesis. Interestingly, resistance to colistin was also observed in one untreated rabbit. As crossresistance to colistin (i.e. a cationic peptide) and host antimicrobial cationic peptides has already been described in Gram-negative bacilli, ${ }^{26,38}$ we investigated the co-occurrence of resistance to HDPs among those strains. The data showed that, in contrast to previous findings, ${ }^{26}$ some colistin-resistant strains isolated in animals with osteomyelitis 24 days post-infection have slightly increased resistance to LL-37, which was enhanced after colistin treatment. This adaptation to the local host environment has already been described with staphylococci in our PJI model. ${ }^{19,20}$ The spontaneous emergence of resistance to AMPs could play a role in PJI persistence, as HDPs are the first line of host defence against pathogens. ${ }^{39}$ This cross-resistance may contribute to the emergence of colistin resistance in patients treated with colistin, as colistin exposure amplifies this phenomenon.

Our study has limitations. First, we used a low dose of meropenem $(80 \mathrm{mg} / \mathrm{kg}$ im q8h, equivalent to only $250 \mathrm{mg}$ iv $\mathrm{q} 8 \mathrm{~h}$ in humans) due to poor tolerability of higher doses in rabbits. Hence, we could not replicate pharmacokinetic/pharmacodynamic targets for meropenem in humans as initially planned and the efficacy of meropenem in vivo may have been underestimated. As meropenem enhances the efficacy of colistin in vivo at this suboptimal dosage, this limitation strengthens the potential interest of this combination, as meropenem doses used in humans are much higher. The second major limitation of our study is that we used only a single strain. Hence, our findings may not be extrapolated to any CPE, especially strains with penem MICs $>8 \mathrm{mg} / \mathrm{L}$.

In conclusion, our findings highlight that colistin monotherapy should not be recommended for treating KPC-producing Enterobacteriaceae osteomyelitis owing to its poor in vivo efficacy and the emergence of resistant strains. For strains with MIC $\leq 8 \mathrm{mg} / \mathrm{L}$, combinations of colistin and meropenem, with or without gentamicin, could be effective, but did not completely prevent the emergence of colistin-resistant strains. The combination of 
colistin and fosfomycin appears promising and should be further evaluated in vivo. The combination of colistin and tigecycline should be administered with caution, as it may be antagonistic in vitro and in vivo. Experimental stringent models such as bone and joint infections may improve our knowledge for designing optimal treatment of CPE, including KPC-producing Enterobacteriaceae, and better inform clinical recommendations. The concordance between in vitro and in vivo bactericidal activity may be used as a tool to screen optimal combinations for selected clinical isolates and design individualized regimens for the treatment of difficult-to-treat CPE infections.

\section{Acknowledgements}

We would like to thank Romain Guilhaumou and Natacha Doudka, Department of Clinical Pharmacology of La Timone Hospital, Marseille, France, for the measurement of colistin plasma levels in rabbits.

\section{Funding} Agency for Drug Safety [Agence Nationale de Sécurité du Médicament (ANSM)] as part of the EVERGREEN study (number AAP-2013-055).

\section{Transparency declarations}

None to declare.

\section{Supplementary data}

Table $\mathrm{S} 1$ is available

\section{References}

1 Shrivastava S, Shrivastava P, Ramasamy J. World Health Organization releases global priority list of antibiotic-resistant bacteria to guide research, discovery, and development of new antibiotics. J Med Soc 2018; 32: 76-7.

2 Cristina ML, Sartini M, Ottria G et al. Epidemiology and biomolecular characterization of carbapenem-resistant Klebsiella pneumoniae in an Italian hospital. J Prev Med Hyg 2016; 57: E149-56.

3 de Sanctis J, Teixeira L, van Duin D et al. Complex prosthetic joint infections due to carbapenemase-producing Klebsiella pneumoniae: a unique challenge in the era of untreatable infections. Int J Infect Dis 2014; 25: 73-8.

4 Abboud CS, Monteiro J, Stryjewski ME et al. Post-surgical mediastinitis due to carbapenem-resistant Enterobacteriaceae: clinical, epidemiological and survival characteristics. Int J Antimicrob Agents 2016; 47: 386-90.

5 Mayberry-Carson KJ, Tober-Meyer B, Smith JK et al. Bacterial adherence and glycocalyx formation in osteomyelitis experimentally induced with Staphylococcus aureus. Infect Immun 1984; 43: 825-33.

6 Crémieux AC, Carbon C. Experimental models of bone and prosthetic joint infections. Clin Infect Dis 1997; 25: 1295-302.

7 Karaiskos I, Souli M, Galani I et al. Colistin: still a lifesaver for the 21st century? Expert Opin Drug Metab Toxicol 2017; 13: 59-71.

8 Grundmann H, Glasner C, Albiger B et al. Occurrence of carbapenemaseproducing Klebsiella pneumoniae and Escherichia coli in the European survey of carbapenemase-producing Enterobacteriaceae (EuSCAPE): a prospective, multinational study. Lancet Infect Dis 2017; 17: 153-63.
9 Macesic N, Nelson B, Uhlemann A-C. Colistin resistance in carbapenemresistant Klebsiella pneumoniae: de novo or drug exposure? Clin Infect Dis 2017; 65: 702-3.

10 Corona A, Cattaneo D. Dosing colistin properly: let's save 'our last resort old drug!'. Clin Infect Dis 2017; 65: 870.

11 Choi M-J, Ko KS. Mutant prevention concentrations of colistin for Acinetobacter baumannii, Pseudomonas aeruginosa and Klebsiella pneumoniae clinical isolates. J Antimicrob Chemother 2014; 69: 275-7.

12 Chen L, Todd R, Kiehlbauch J et al. Notes from the field: pan-resistant New Delhi metallo- $\beta$-lactamase-producing Klebsiella pneumoniae - Washoe County, Nevada, 2016. MMWR Morb Mortal Wkly Rep 2017; 66: 33.

13 Bassetti M, Giacobbe DR, Giamarellou H et al. Management of KPCproducing Klebsiella pneumoniae infections. Clin Microbiol Infect 2018; 24: 133-44.

14 Rodríguez-Baño J, Cisneros JM, Gudiol C et al. Treatment of infections caused by carbapenemase-producing Enterobacteriaceae. Enferm Infecc Microbiol Clin 2014; 32 Suppl 4: 49-55.

15 Crandon JL, Nicolau DP. Human simulated studies of aztreonam and aztreonam-avibactam to evaluate activity against challenging Gramnegative organisms, including metallo- $\beta$-lactamase producers. Antimicrob Agents Chemother 2013; 57: 3299-306.

16 Mimoz O, Grégoire N, Poirel L et al. Broad-spectrum $\beta$-lactam antibiotics for treating experimental peritonitis in mice due to Klebsiella pneumoniae producing the carbapenemase OXA-48. Antimicrob Agents Chemother 2012; 56: $2759-60$

17 Wiskirchen DE, Nordmann P, Crandon JL et al. Efficacy of humanized carbapenem exposures against New Delhi metallo- $\beta$-lactamase (NDM-1)-producing Enterobacteriaceae in a murine infection model. Antimicrob Agents Chemother 2013; 57: 3936-40.

18 Napier BA, Burd EM, Satola SW et al. Clinical use of colistin induces crossresistance to host antimicrobials in Acinetobacter baumannii. MBio 2013; 4: e00021-13.

19 Mishra NN, Yang S-J, Chen L et al. Emergence of daptomycin resistance in daptomycin-naive rabbits with methicillin-resistant Staphylococcus aureus prosthetic joint infection is associated with resistance to host defense cationic peptides and mprF polymorphisms. PLoS One 2013; 8: e71151.

20 Saleh-Mghir A, Muller-Serieys C, Dinh A et al. Adjunctive rifampin is crucial to optimizing daptomycin efficacy against rabbit prosthetic joint infection due to methicillin-resistant Staphylococcus aureus. Antimicrob Agents Chemother 2011; 55: 4589-93.

21 Belmatoug N, Crémieux AC, Bleton R et al. A new model of experimental prosthetic joint infection due to methicillin-resistant Staphylococcus aureus: a microbiologic, histopathologic, and magnetic resonance imaging characterization. J Infect. Dis 1996; 174: 414-7.

22 Courvalin P. Bactericidie: Aspects Theoriques et Therapeutiques. Paris, France: Editions Maloine, 1991.

23 Balouiri M, Sadiki M, Ibnsouda SK. Methods for in vitro evaluating antimicrobial activity: a review. J Pharm Anal 2016; 6: 71-9.

24 Pournaras S, Vrioni G, Neou E et al. Activity of tigecycline alone and in combination with colistin and meropenem against Klebsiella pneumoniae carbapenemase (KPC)-producing Enterobacteriaceae strains by time-kill assay. Int J Antimicrob Agents 2011; 37: 244-7.

25 Norden CW. Experimental osteomyelitis. I. A description of the model. J Infect Dis 1970; 122: 410-8.

26 Dobias J, Poirel L, Nordmann P. Cross-resistance to human cationic antimicrobial peptides and to polymyxins mediated by the plasmid-encoded MCR-1? Clin Microbiol Infect 2017; 23: 676.e1-5.

27 Poirel L, Jayol A, Bontron $S$ et al. The mgrB gene as a key target for acquired resistance to colistin in Klebsiella pneumoniae. J Antimicrob Chemother 2015; 70: 75-80. 
28 Hancock RE, Diamond G. The role of cationic antimicrobial peptides in innate host defences. Trends Microbiol 2000; 8: 402-10.

29 Andersson DI, Hughes D, Kubicek-Sutherland JZ. Mechanisms and consequences of bacterial resistance to antimicrobial peptides. Drug Resist Updat 2016; 26: 43-57.

30 Development Core Team R. R: A Language and Environment for Statistical Computing. Vienna, Austria: R Foundation for Statistical Computing, 2011.

31 Hothorn T, Bretz F, Westfall P. Simultaneous inference in general parametric models. Biom J 2008; 50: 346-63.

32 Zhao M, Bulman ZP, Lenhard JR et al. Pharmacodynamics of colistin and fosfomycin: a 'treasure trove' combination combats KPC-producing Klebsiella pneumoniae. J Antimicrob Chemother 2017; 72: 1985-90.

33 Bassetti M, Peghin M, Pecori D. The management of multidrug-resistant Enterobacteriaceae. Curr Opin Infect Dis 2016; 29: 583-94.

34 Albur M, Noel A, Bowker $K$ et al. Bactericidal activity of multiple combinations of tigecycline and colistin against NDM-1-producing Enterobacteriaceae. Antimicrob Agents Chemother 2012; 56: 3441-3.
35 Michail G, Labrou M, Pitiriga V et al. Activity of tigecycline in combination with colistin, meropenem, rifampin, or gentamicin against KPC-producing Enterobacteriaceae in a murine thigh infection model. Antimicrob Agents Chemother 2013; 57: 6028-33.

36 Corvec S, Furustrand Tafin U, Betrisey B et al. Activities of fosfomycin, tigecycline, colistin, and gentamicin against extended-spectrum- $\beta$-lactamaseproducing Escherichia coli in a foreign-body infection model. Antimicrob Agents Chemother 2013; 57: 1421-7.

37 Zimmerli W, Frei R, Widmer AF et al. Microbiological tests to predict treatment outcome in experimental device-related infections due to Staphylococcus aureus. J Antimicrob Chemother 1994; 33: 959-67.

38 Llobet E, Martínez-Moliner V, Moranta D et al. Deciphering tissue-induced Klebsiella pneumoniae lipid A structure. Proc Natl Acad Sci USA 2015; 112: E6369-78.

39 Koprivnjak T, Peschel A. Bacterial resistance mechanisms against host defense peptides. Cell Mol Life Sci 2011; 68: 2243-54. 